\title{
Die neuromuskuläre Steuerung des Kaumechanismus und ihre Bedeutung für die Kieferorthopädie
}

\section{Neuromuscular Control of the Masticatory Mechanism and its Impact on Orthodontics}

Autoren

J. Fanghänel, C. Kirschneck

Institut

Poliklinik für Kieferorthopädie, Universitätsklinikum

Regensburg

Schlüsselwörter

Kaumechanismus, neuromuskuläre Steuerung

Key words

mastricatory mechanism, neuromuscular control

Bibliografie

DOI https://doi.org/10.1055/a-1108-6775

Inf Orthod Kieferorthop 2020; 52: 91-96

(c) Georg Thieme Verlag KG Stuttgart · New York ISSN 0020-0336

Korrespondenzadresse

Prof. Dr. med. Jochen Fanghänel

Poliklinik für Kieferorthopädie

Universitätsklinikum Regensburg

Franz-Josef-Strauß-Allee 11

D-93053 Regensburg

Tel.: + 49941944 4990, Fax.: + 499419446169

jochen.fanghaenel@ukr.de

\section{ZUSAMMENFASSUNG}

Der Kaumechanismus gehört zu den kompliziertesten neuromuskulären Leistungen des menschlichen Organismus. Er setzt ein komplexes biologisches System mit einer optimalen multifaktoriellen Steuerung voraus. Dabei ist eine effektive afferente und efferente Innervation aller am Kaumechanismus beteiligten Strukturen sowie ihre optimale Koordination im Sinne eines neuromuskulären Regelkreises erforderlich. Der geregelte Ablauf der Kieferbewegungen wird durch neuronale Regelzyklen unter der Kontrolle des Cortex cerebri und subkortikaler Areale gesteuert. Dabei laufen alle Vorgänge der Steuerung in 3 Ebenen ab. In der lokalen Ebene registrieren Rezeptoren die lokale Situation, in der (sub)kortikalen Ebene werden Erregungen in Großhirnrinde und Basalganglien verarbeitet sowie die (extra)pyramidalen Bahnen formiert. Schließlich werden alle am Kauakt beteiligten Muskeln aktiviert. Fehlerhafte oder traumatische Okklusionskontakte, wie sie im Rahmen von Malokklusionen und bei erwachsenen Patienten im parodontal vorgeschädigten Gebiss auftreten, haben dabei das Potenzial, den neuromuskulären Regelkreis des stomatognathen Systems empfindlich zu verändern und Fehlregulationen auszulösen. Eine genaue Kenntnis der im Rahmen des Artikels besprochenen neuromuskulären Steuerung des Kaumechanismus ist daher für den Kieferorthopäden, der therapeutisch in dieses System eingreift, von großer Bedeutung.

\section{ABSTRACT}

The chewing mechanism is one of the most complicated neuromuscular functions in the human body. It requires a complex biological system with optimal multifactorial control. This requires effective afferent and efferent innervation of all structures involved in the chewing mechanism and their optimal coordination in the sense of a neuromuscular control loop. The regulated sequence of jaw movements is controlled by neuronal control cycles under the control of the cortex and subcortical areas. All control processes run on 3 levels. At the local level, receptors register the local situation, at the (sub)cortical level excitations are processed in the cerebral cortex and basal ganglia and (extra)pyramidal pathways are formed. Finally, all muscles involved in the chewing act are activated. Faulty or traumatic occlusal contacts, which occur in the context of malocclusions and in the periodontically damaged dentition in adult patients, have the potential to sensitively alter the neuromuscular control loop of the stomatognathic system and trigger malregulations. A profound knowledge of the neuromuscular control of the chewing mechanism discussed in this article is therefore of major importance for the orthodontist, who therapeutically intervenes in this system. 


\section{Einführung}

Das Ziel einer jeden kieferorthopädischen Therapie ist die Rehabilitation des stomatognathen Systems in funktioneller und ästhetischer Hinsicht. Eine der wichtigsten Funktionen stellt dabei der Kaumechanismus dar, welcher einer komplexen und abgestimmten neuromuskulären Steuerung unterliegt. Unzählige Mechanorezeptoren v. a. im Parodont, Kiefergelenk und der orofazialen Muskulatur können dabei feinste Störungen in der Okklusion detektieren, was zu Fehlregulationen bzw. -belastungen führen kann, von denen angenommen wird, dass sie langfristig auch zu Schäden im stomatognathen System bzw. Kiefergelenk führen können. Insbesondere traumatische Okklusionskontakte, wie sie häufig bei erwachsenen Patienten im parodontal vorgeschädigten Gebiss mit pathologischen Zahnwanderung auftreten [1, 2], aber auch ausgeprägte Malokklusionen können eine empfindliche Störgröße für das stomatognathe System ( $\triangleright$ Abb. 1) darstellen, welche je nach Plastizität und Anpassungsfähigkeit der neuronalen Strukturen zu funktionellen Problemen und Störungen beim Kauen führen können sowie zu Jiggling-Effekten an den Zähnen mit Progression parodontaler Knochenverluste [1,2]. Die orthodontische Therapie bietet nun die Möglichkeit, die Okklusion des Patienten dementsprechend zu verändern und traumatische Okklusionskontakte zu eliminieren, greift aber dadurch auch maßgeblich in die neuromuskuläre Steuerung durch Veränderung der okklusalen Belastungssituation dar. Sie kann daher präventiv-therapeutisch bezüglich Fehlregulationen wirksam werden. In diesem Zusammenhang wird in der Literatur auch eine Entstehung und Progression von kraniomandibulären Dysfunktionen diskutiert. Die derzeitige Evidenz deutet jedoch darauf hin, dass kieferorthopädische Maßnahmen und damit eine diesbezügliche Veränderung der Okklusion der Patienten „CMD-neutral“ sind, d. h. kraniomandibuläre Dysfunktionen weder verursachen noch präventiv-kurativ diesbezüglich wirken können [3,4]. Aufgrund der Bedeutung von Okklusionsveränderungen und damit des mechanosensitiven Inputs für die komplexen Vorgänge der neuromuskulären Steuerung des Kaumechanismus sollen diese im Folgenden näher erläutert werden, um kieferorthopädische Maßnahmen in diesem Kontext besser einordnen zu können.

\section{Allgemeine Betrachtungen zum Kaumechanismus}

Das stomatognathe System, dessen Strukturen den Kaumechanismus und die neuromuskuläre Steuerung vollziehen, ist ein aus zahlreichen Bestandteilen zusammengesetzter Funktionskreis ( $\mathbf{A} \mathbf{b b} \mathbf{b} \mathbf{1}$ ). Entwicklungsgeschichtlich ist dieses System von den Strukturen der Kiemenbogenregion abzuleiten. In ihm nehmen das Kiefergelenk und die Zähne eine zentrale Stellung ein. Dabei zeigt die Muskulatur eine sehr enge Abstimmung mit den Formmerkmalen einschließlich der individuellen Zahnstellung [5]. Die Steuerung aller Unterkieferbewegungen, welche zur Ausübung mastikatorischer (und auch phonetischer) Funktionen erforderlich sind, unterliegt einem komplexen Reflexmechanismus, der mit einem kybernetischen System vergleichbar ist. Durch diesen sind fein abgestimmte Unterkiefer- und Zungenbewegungen, die Einstellung der Kaukraft, die quantitative und qualitative Abgabe von Speichel (Para-

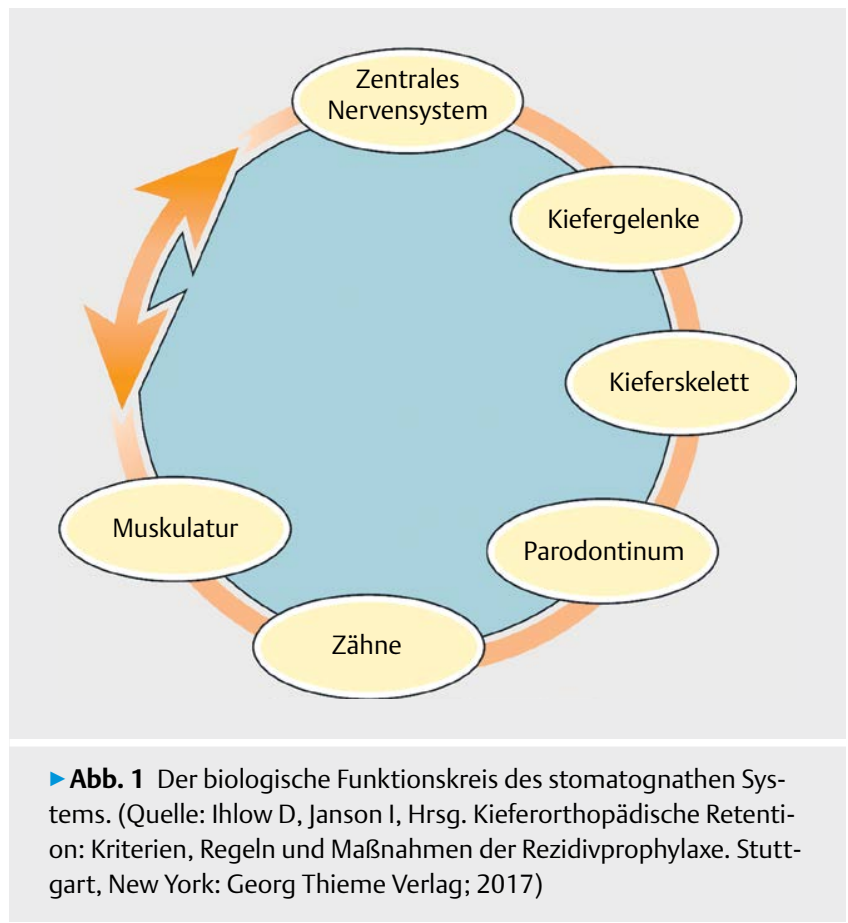

sympathikus), die Abstimmung der Mandibulabewegungen (sog. Slow-Reflex-Zyklen) [6], die Aktivierung der Sinnesorgane u.a.m. sowie auch die Koordination aller genannten Funktionen möglich. Außerdem können Willensimpulse in diesen Kaumechanismus eingreifen und die Kiefer- und Zungenbewegungen sowie die Kaukraft beeinflussen [7]. Mit zunehmendem Alter ist eine Umstellung dieses Reflexmechanismus und beispielsweise die Anpassungsfähigkeit an Prothesen erschwert bzw. verringert. Besondere Ausprägungen und Veränderungen der oben genannten komplexen Mechanismen finden wir bei Apoplexie, Hypertonus, Krampfleiden und anderen Erkrankungen des Nervensystems wie epileptischen Anfällen, Parkinsonismus und Veitstanz.

\section{Zum Kaumechanismus und seiner Steuerung}

Die Abläufe der neuromuskulären Steuerung vollziehen sich in 3 Ebenen ( $>$ Abb. 2). Dabei sind die jeweiligen Strukturen in die erforderlichen Afferenzen (Input) und Efferenzen (Output) des Zentralnervensystems eingebettet. Die Vielzahl und Kompliziertheit der neuronalen Regelkreise bewirkt die Abgestimmtheit, Zielsicherheit und Zweckmäßigkeit des Bewegungsablaufs sowie die Einstellung der entsprechenden erforderlichen Kaukraft. Bei den Mandibulabewegungen bedingen willkürliche (pyramidale Bahnen) und unwillkürliche (extrapyramidale Bahnen) Erregungen einander [8] ( Abb. 2). Das gesamte System wird vom Kauzentrum in der Pons gesteuert und kontrolliert. Das Kleinhirn ist dabei ein Integrationszentrum für die Erhaltung des Gleichgewichts, die Bewegungskoordination und die Regulation des Muskeltonus.

\section{Lokale Ebene}

Die erforderlichen Erregungen aus der Peripherie werden über Neurone der Hirnnerven V2-3, IX und X in die Hirnrinde des ZNS gelei- 


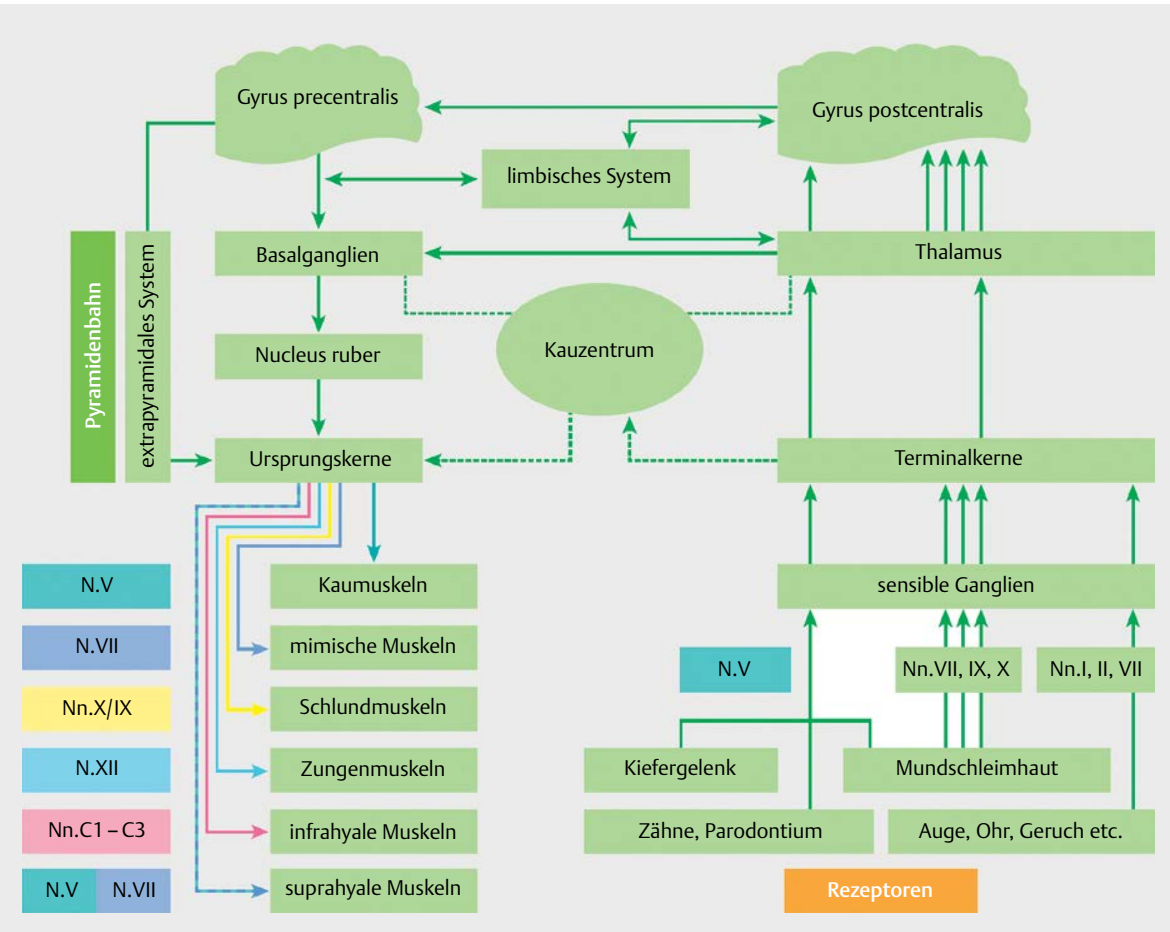

Abb. 2 Der Regelkreislauf zur neuromuskulären Steuerung des Kaumechanismus. (Quelle: Fanghänel J, Proff P, Behr M, Kirschneck C. Kapitel 6 - Wie wird das kraniomandibuläre System neuromuskulär gesteuert? In: Behr M, Fanghänel J, Hrsg. Kraniomandibuläre Dysfunktionen: Antworten auf Fragen aus der Praxis. Stuttgart, New York: Georg Thieme Verlag; 2019: 62-67).

tet ( $>$ Abb. 2). Die Reizaufnahme erfolgt durch verschiedene Rezeptoren (Sensoren), welche im stomatognathen System vielerorts lokalisiert sind: auf der Gesichtshaut, Mundschleimhaut, in den Dentinkanälen (Odontoblastenfortsätze), im Parodontium, in der Kiefergelenkkapsel, in den Muskeln und in der Zunge. Freie Nervenendigungen liegen in allen Bereichen des stomatognathen Systems [9-11]. Bei den Rezeptoren handelt es sich um Strukturen, welche verschiedene Reize wie Berührung, Kraft, Druck, Schmerz, Temperatur, Licht sowie akustische und gustatorische Rezeptionen in elektrische Aktionspotenziale umwandeln, welche über entsprechende Nerven dem zentralen Nervensystem zugeführt werden. Im stomatognathen System finden wir vor allem sogenannte Mechanorezeptoren, zu denen Vater-Pacini-Körperchen ( $>$ Abb. 3a/b), Ruffini-Kör-

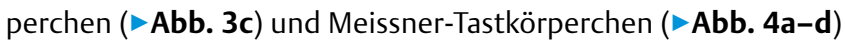
zählen. Sie nehmen hauptsächlich Berührung, Schwerkraft, Druck sowie Beschleunigung wahr.

Vater-Pacini-Körperchen haben einen lamellenförmigen Aufbau. Im Zentrum eines Körperchens befindet sich ein Dendritenende, welches zwiebelschalenartig von Gliazellen und außen von einer Schicht Perineuralzellen umgeben ist $[9,10]$. Ruffini-Körperchen haben eine gering lamellierte Kapsel, welche einen flüssigkeitserfüllten Raum umschließt. Der Raum ist von funktionell spezialisierten Kollagenfasern durchzogen. Diese treten mit Kollagenfasern in der Umgebung der Körperchen in Verbindung [9, 10]. Meissner-Tastkörpcherchen befinden sich in der Papillarschicht der Dermis. Diese sind durch kurze Kollagenfasern mit der Basallamina verbunden. Sie bestehen aus aufgeknäulten Dendriten. Lamellen- artige Fortsätze von Gliazellen umgeben diese Knäuel. Lediglich der basale Abschnitt dieser Struktur hat eine Kapsel.

Die freien Nervenendigungen, Nozizeptoren und Nozisensoren ( $\triangleright$ Abb. 5) stellen das terminale Ende eines myelinisierten bzw. nichtmyelinisierten Rezeptoraxons dar. Es sind Endstrukturen von A $\delta$ - und C-Fasern. Ihre Reizung führt zu Schmerzempfindungen. In den Auftreibungen, welche nicht von Schwann-Zellen bedeckt sind, befinden sich zahlreiche Vesikel und Mitochondrien. Zur Nozizeption führen mechanische, thermische und chemische Reize, die auch kombiniert sein können.

Die afferenten Neurone aller oben genannten Rezeptoren gelangen zu den Terminalkernen der entsprechenden Hirnnerven im Rauten- und Mittelhirn. Von hier führen sie zum Thalamus als Zentrum der Sensibilität ( $>$ Abb. 2 und 6). Der Thalamus erhält ebenfalls Erregungen durch psychische Empfindungen aus den Sinnesorgangen über die Hirnnerven I, II und VIII. Es bestehen aber auch Verbindungen mit dem limbischen System ( $\bullet$ Abb. 2), welches wesentliche Strukturen für Emotionen, Antrieb und Gedächtnis besitzt.

\section{Kortikale und subkortikale Ebene}

Nachdem die Umschaltung der Erregungen im Thalamus erfolgen, gelangen diese zum und hinter den Gyrus postcentralis des Großhirns (\Abb. 2) (Körperfühlsphäre, primäres somato-sensibles Rindenfeld). Diese Bahnen sind ein Teil der sogenannten Thalamusstrahlung (>Abb. 6). Bahnen gelangen auch vom Thalamus zu den Basalganglien (subkortikale Ebene) (> Abb. 2). Von der Körperfühl- 

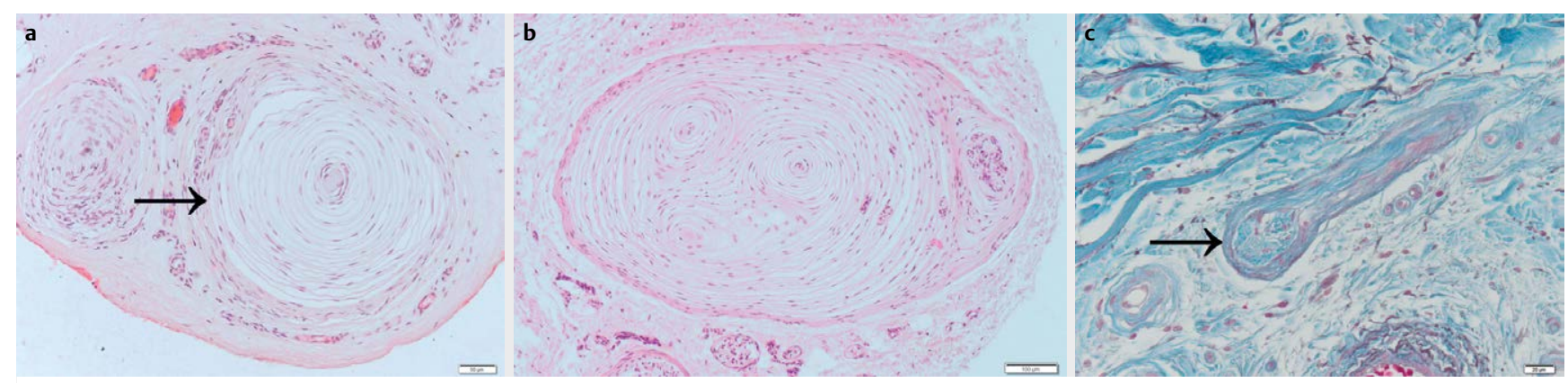

Abb. 3 Mechanorezeptoren im stomatognathen System. a-b Vater-Pacini-Lamellenkörperchen (Hämatoxylin-Eosin). c Ruffini-Körperchen (Kresazan). Quelle: Dr. B. Miehe, Institut für Anatomie und Zellbiologie, Universitätsmedizin Greifswald.

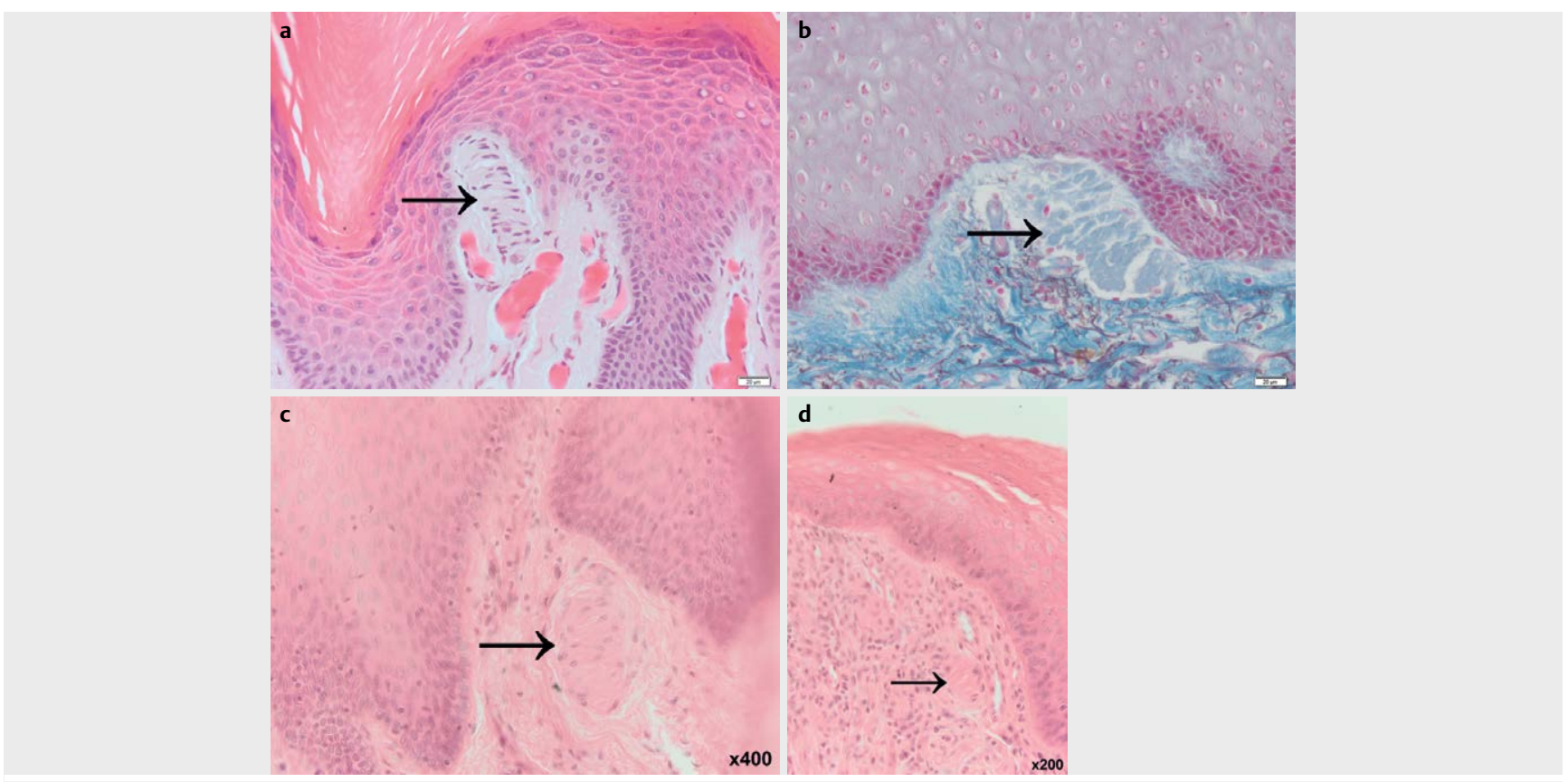

Abb. 4 Meissner-Tastkörperchen (a, c, d Hämatoxylin-Eosin, b Kresazan). Quelle: Dr. B. Miehe, Institut für Anatomie und Zellbiologie, Universitätsmedizin.

sphäre erreichen die Erregungen den Gyrus praecentralis (primäres motorisches Rindenfeld, Motocortex). Das primäre motorische Rindenfeld ist die oberste „Zentrale für die Bewegungsführung“ [12]. Hier beginnen die Pyramidenbahnen (Tractus corticospinales), mit welchen wir willkürliche Bewegungen durchführen können ( $\triangleright$ Abb. 2). Sie wirken modifizierend, dämpfend, hemmend und bewirken eine Zielsicherheit der Bewegung [13]. Die Basalganglien, auf welche die Erregungen vom Thalamus oder von der Großhirnrinde her umgeschaltet werden, gehören zum extrapyramidalen System. Sie bilden den Ursprung der extrapyramidalen Bahnen ( Abb. 2). Diese sind für die Einleitung und Durchführung unbewusster, langsamer, rampenförmiger Bewegungen sowie für die zeitliche und räumliche Koordination der Muskeltätigkeiten verantwortlich [12, 14, 15]. Von den Basalganglien ( die Erregungen u. a. über den Nucleus ruber und die Formatio reticularis zu den Ursprungskernen der entsprechenden Hirn- und Zervikalnerven geleitet. Mit diesen Bahnen erreichen letztlich die
Erregungen die motorischen Endplatten aller an der Mandibulabewegung beteiligten Muskeln. Das extrapyramidale System ist mit einem „Servomechanismus“ vergleichbar, welcher selbständig Exkursionen mit unterstützt [13].

Über das oben genannte Pyramidenbahnsystem „kontrolliert“ der Kortex die extrapyramidalen motorischen Zentren. Einerseits können die Fasern dämpfend und hemmend wirken, andererseits gehen von ihnen aber auch ständig tonische Erregungen aus. Die automatischen und stereotypen Mandibulabewegungen, welche von den subkortikalen Zentren initiiert werden, sollen durch den Einfluss pyramidaler Reize modifiziert werden, sodass daraus gezielte, fein abgestimmte Bewegungen resultieren. Deshalb ist es von sehr großer Bedeutung, dass pyramidale und extrapyramidale Bahnen miteinander gekoppelt sind [15]. Diese Kopplung trägt wesentlich zur Zielsicherheit des Bewegungsablaufs bei. 


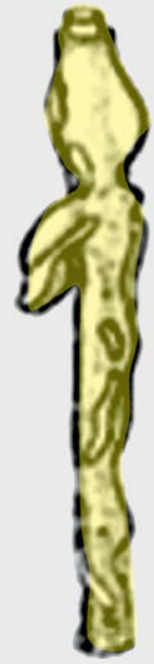

A $\delta$

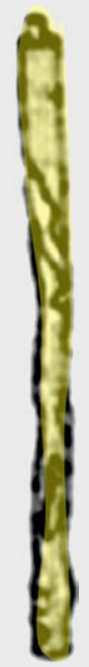

C
- Abb. 5 Freie Nervenendigungen bzw. Nozizeptoren und Nozisensoren von einem Aס-Nerv und einem C-Nerv.

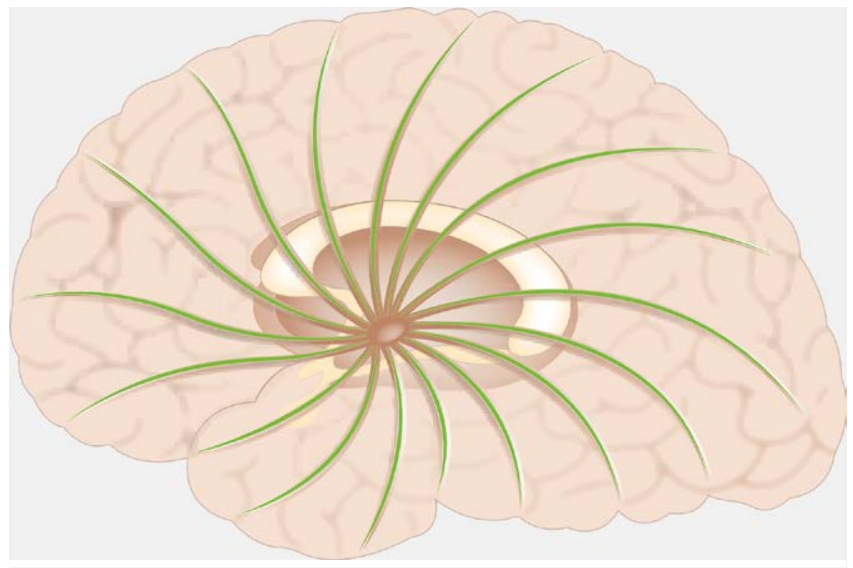

- Abb. 6 Der Thalamus als Zentrum der Sensibilität des Körpers. Die „Thalamusstrahlung" stellt das dritte Neuron eines afferenten Nerven dar. (Quelle: Behr M, Fanghänel J, Hrsg. Kraniomandibuläre Dysfunktionen: Antworten auf Fragen aus der Praxis. Stuttgart, New York: Georg Thieme Verlag; 2019).

\section{Lokale Ebene - Ende des Regelkreises}

Diese Ebene wird repräsentiert durch alle am Kaumechanismus beteiligten Muskeln ( $\triangleright$ Abb. 2 und 7). Diese sind [8, 11]:

- eigentliche Kaumuskeln: M. temporalis, M. masseter, M. pterygoideus medialis, M. pterygoideus lateralis

- Kauhilfsmuskeln - suprahyoidale Muskulatur (Mundbodenmuskeln): M. mylohyoideus, M genioglossus, M. digastricus (Venter anterior und posterior), M. stylohyoideus

- Kauhilfsmuskeln - infrahyoidale Muskulatur: M. thyrohyoideus, M. sternohyoideus, M. sternothyroideus, M. omohyoideus
" „indirekte Kaumuskeln“ (Nackenmuskeln) als „Widerlager“ und Antagonisten der Kauhilfsmuskeln. Muskeln, die in erster Linie für den Transport der Nahrung in den Pharynx verantwortlich sind: Zungen-, Gaumen- und Pharynxmuskulatur

Aus der Vielzahl der beteiligten Muskeln und ihrer Innervation ( Abb. 2) können wir die Komplexizität des Kaumechanismus erkennen [11]. Alle Muskeln folgen dem „Alles-oder-Nichts-Prinzip“. Sie gehören dem „Twitch“-Typ an und können u. a. durch molekularbiologische Darstellung der myofibrillären ATPase anhand der Succinatdehydrogenase klassifiziert werden. Es handelt sich um eine Mitochondriendarstellung. Wir unterscheiden dabei:

- Typ I: langsame Fasern, ATPase-arm

- Typ lla: ausdauernde, kurzfristige intensive Fasern, ATPase-reich

- Typ Ilb: schnelle Fasern, ATPase-reich

Jeder Muskel besitzt alle 3 Fasertypen. Durch diese Faserkombination entsteht ein Muskelspiel, welches den Anforderungen und letztlich auch der Feinabstimmung gerecht wird.

\section{Zur Rolle des Kleinhirns}

Die Kleinhirnkerne liegen im Nebenschluss der pyramidalen und extrapyramidalen Bahnen [9]. Hierarchisch gesehen sind diese Kerne Zentren, welche an der Programmierung kortikal induzierter Bewegungsabläufe beteiligt sind [16]. Diese stellen Integrationszentren für die Gleichgewichtserhaltung und Bewegungskoordination dar. Sie sind des Weiteren für die Regulierung des Muskeltonus mit verantwortlich. Ausfälle bzw. Schäden durch hohen Alkoholkonsum haben hochgradige Störungen der Bewegungskoordination $[6,16]$ zur Folge. Des Weiteren ist das Kleinhirn für die Programmierung rascher Bewegungen und deren Kurskorrekturen zuständig.

\section{Einschränkungen der neuromuskulären Steuerung}

Um ein künstliches Kiefergelenk zu implantieren, ist es notwendig, Mandibula und Os temporale teilweise zu resezieren ( $>$ Abb. 8). Damit wird auch Bindegewebe und ebenfalls das Kiefergelenk mit den entsprechenden Rezeptoren wegfallen. Dabei fehlen Afferenzen und eine Feinabstimmung in der neuromuskulären Steuerung ist nicht mehr möglich [17]. Störungen in der neuromuskulären Steuerung finden wir auch bei dentalen Implantaten aufgrund des entsprechenden fehlenden Parodonts (Wegfall von Rezeptoren) [17]. Sensible und motorische Störungen treten bei Trigeminusund Glossopharyngeusneuralgien auf, die auch den Kaumechanismus beeinflussen [18]. Stresssituationen und Fehlregulationen des limbischen Systems gehen mit Spasmen der Kaumuskulatur und schlecht lokalisierbaren Gesichtsschmerzen einher. Diese Symptome beeinflussen negativ den Kaumechanismus [7].

\section{Interessenkonflikt}

Die Autoren geben an, dass kein Interessenkonflikt besteht. 


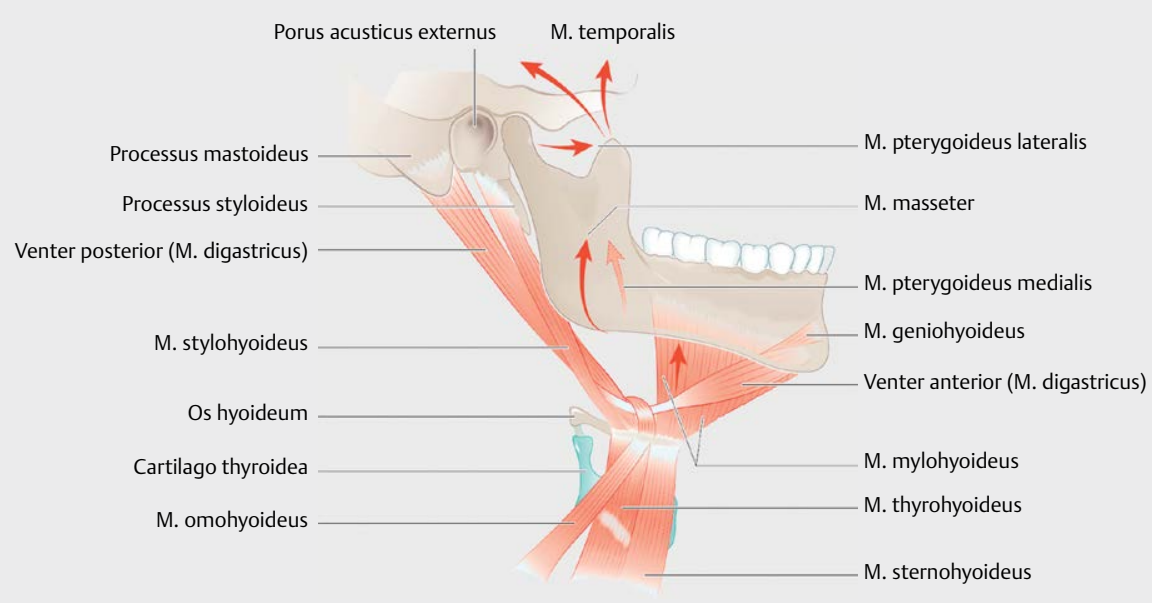

Abb. 7 Das Zusammenspiel von Kaumuskulatur sowie supra- und infrahyoidaler Muskulatur bei Kaubewegungen. Die roten Pfeile geben die Zugrichtungen der Muskeln an. (Quelle: Behr M, Fanghänel J Hrsg. Kraniomandibuläre Dysfunktionen: Antworten auf Fragen aus der Praxis. Stuttgart, New York: Georg Thieme Verlag; 2019).

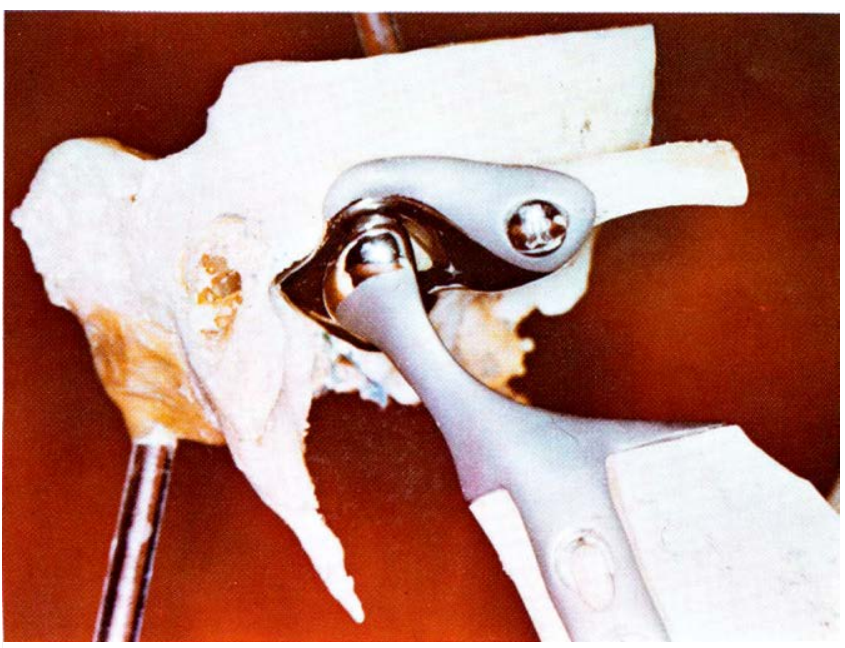

Abb. 8 Künstliches Kiefergelenk. Totalprothese am Modell (Chrom-Kobalt-Molybdän-Legierung). (Quelle: Fanghänel J, Proff P, Behr M, Kirschneck C. Kapitel 6 - Wie wird das kraniomandibuläre System neuromuskulär gesteuert? In: Behr M, Fanghänel J, Hrsg. Kraniomandibuläre Dysfunktionen: Antworten auf Fragen aus der Praxis. Stuttgart, New York: Georg Thieme Verlag; 2019: 62-67).

\section{Literatur}

[1] Kirschneck C, Proff P. Orthodontic Treatment in the Periodontally Damaged Dentition - Advantages, Possibilities and Limitations. Inf Orthod Kieferorthop 2016; 48: 79-86

[2] Kirschneck C. Kieferorthopädie im parodontal geschädigten Gebiss Schnittstelle zwischen Kieferorthopädie und Parodontologie. Quintessenz 2017; 68: 533-544

[3] Kirschneck C, Proff P. Kapitel 15 - Welche wechselseitigen Beziehungen bestehen zwischen kieferorthopädischer Therapie und kraniomandibulären Dysfunktionen? In: Behr M, Fanghänel J, Hrsg. Kraniomandibuläre Dysfunktionen: Antworten auf Fragen aus der Praxis. Stuttgart, New York: Georg Thieme Verlag; 2019: 122-127
[4] Kirschneck C. Dysgnathie, Kieferorthopädie und kraniomandibuläre Dysfunktion - gibt es Zusammenhänge? Quintessenz 2017; 68: $1253-1260$

[5] Fanghänel J, Gedrange T, Proff P. The face-physiognomic expressiveness and human identity. Ann Anat 2006; 188: 261-266

[6] Kubein-Meesenburg D, Nägerl H, Fanghänel J. Biomechanik und neuromuskuläre Steuerung des Kiefergelenkes. In: Benner K-U, Fanghänel J, Kowalewski R, u. a. Hrsg. Morphologie, Funktion und Klinik des Kiefergelenks. Berlin, New York: Quintessenz; 1993: 61-100

[7] Schumacher G-H. Anatomie des Kiefer-Gesichts-Bereiches: Für den Zahntechniker und die Zahnarzthelferin. 4 Aufl. Berlin: Ullstein Mosby; 1993

[8] Behr M, Fanghänel J, Hrsg. Kraniomandibuläre Dysfunktionen: Antworten auf Fragen aus der Praxis. Stuttgart, New York: Georg Thieme Verlag; 2019

[9] Samandari F, Mai JK Curriculum Funktionelle Anatomie für Zahnmediziner. 2 Aufl. Berlin, Chicago, London: et al. Quintessenz; 2009

[10] Junqueira LC, Carneiro J. Basic histology: Text \& Atlas. 10 Aufl. New York: Lange Medical Books McGraw-Hill; 2003

[11] Fanghänel J, Pera F, Anderhuber F et al. Waldeyer Anatomie des Menschen. 17 Aufl. Berlin, New York: De Gruyter; 2003

[12] Fanghänel J, Kubein-Meesenburg D, Proff P. Anatomie und funktionelle Darstellung des orofazialen Systems. Quintessenz Zahntechn 2011; 37: $1122-1127$

[13] Schumacher G-H. Anatomie für Zahnmediziner: Lehrbuch und Atlas. 3 AuflHeidelberg: Hüthig; 1997

[14] Schmidt RF, Dudel J, Hrsg. Grundriss der Neurophysiologie. 6 Aufl. Berlin: Springer-Verlag; 1987

[15] Scapino RP. Histopathology associated with malposition of the human temporomandibular joint disc. Oral Surg Oral Med Oral Pathol 1983; 55: 382-397

[16] Ridder P. Craniomandibuläre Dysfunktion: Interdisziplinäre Diagnoseund Behandlungsstrategien. 3 Aufl. München: Urban \& Fischer; 2016

[17] Fanghänel J, Proff P, Behr M et al. Kapitel 6 - Wie wird das kraniomandibuläre System neuromuskulär gesteuert? In: Behr M, Fanghänel J, Hrsg. Kraniomandibuläre Dysfunktionen: Antworten auf Fragen aus der Praxis. Stuttgart, New York: Georg Thieme Verlag; 2019: 62-67

[18] Fanghänel J, Paul I, Meyer FU. Der Schmerz im orofazialen System aus morphologischer Sicht. Dtsch Zahnärztl Z 1996; 51: 382-385 